\title{
Effect of linear polarizers on highly focused spirally polarized fields
}

\author{
Rosario Martínez-Herrero, David Maluenda \\ Universidad Complutense de Madrid, Facultad de Ciencias Físicas, Departamento de \\ Óptica, Ciudad Universitaria s/n, 28040 Madrid (Spain) \\ Ignasi Juvells, Artur Carnicer ${ }^{1}$ \\ Universitat de Barcelona (UB), Facultat de Física, Departament de Física Aplicada, Martí \\ i Franquès 1, 08028 Barcelona (Spain)
}

\begin{abstract}
Linear polarizers are commonly used for projecting the direction of the electric field of a transverse paraxial beam on the direction of the polarizer axis. However, the use of these devices with highly convergent field poses a practical problem because the non transversal character of electric field. In this article, we discuss the behavior of highly focused beams with spiral polarization when they pass through a polarizer. Interestingly, beams with azimuthal polarization display a non negligible irradiance in the direction of propagation after passing through a polarizer. On top of that, we found that the irradiance of a highly focused radially polarized beam after a polarizer is notably different from the projection of the field on the direction of the polarizer axis.
\end{abstract}

Keywords: Physical Optics, Polarization, Highly focused beams 2010 MSC: 78A10, 78A25, 78A45

\section{Introduction}

Polarizers are devices present in almost all optical systems. They are designed to project the electric field in the direction of the polarizer axis and in addition, they can be used to analyze the state of polarization of light. In par5 ticular, it is well known that the Stokes parameters can be determined with the help of a linear polarizer used in combination with a $\lambda / 4$ plate. It is assumed that polarizers are intended for paraxial beams with the electric field vibrating in a plane transverse to the direction of propagation. As some authors have pointed out [1-3], the use of polarizers with highly focused beams raises

\footnotetext{
${ }^{1}$ Corresponding author email: artur.carnicer@ub.edu
} 
doubts about how the beam is modified due to the presence of a non-negligible longitudinal electric field component.

Nowadays, highly focused beams are present in a variety of scenarios and multiples studies and potential applications have been proposed in the literature [4-17]. For this reason, we have recently published a study on how linear

15 polarizers modify a tightly focused field when these devices are placed at the focal plane of a high numerical aperture objective lens [18]. The polarizer was modeled as a plane-parallel uniaxial absorbing medium with the optical axis parallel to the plate surfaces of the polarizer [19-22]. We found that the electric field component in the direction of propagation and the influence of the

20 Fresnel coefficients modify the performance of the polarizer. In particular, it was demonstrated that the Malus' law is not strictly fulfilled for highly focused beams. In the present paper, we focus on analyzing how beams with spiral polarization are modified when they passes through a linear polarizer. The important cases of radial and azimuthal polarization are discussed in depth.

The article is organized as follows. Section 2 reviews basic concepts on propagation of electric fields in the focal area (Richards-Wolf equation). In section 3 , we describe a theoretical model for O-type polarizers and the projection vector equation for the angular spectrum of plane waves are also considered. We also study how the projection of the angular spectrum modifies the components 30 of the electric field after passing though the polarizer. In particular, we focus our attention on spirally, radially and azimuthally polarized beams. In section 4 we discuss several parameters that are useful to provide more insight on the behavior of the beam after crossing the polarizer. Finally, the conclusions are presented in section 5 .

\section{Electromagnetic fields in the focal area}

The Richards-Wolf equation provides the framework to describe convergent electromagnetic beams in the focal region of a a high NA lens. This formula provides a relationship between the transverse illuminating beam $\mathbf{E}_{i}=\left(E_{i x}, E_{i y}, 0\right)$ and the focused field distribution $\mathbf{E}$ [23]:

$$
\mathbf{E}(\mathbf{r})=A \int_{0}^{\theta_{M}} \int_{0}^{2 \pi} \mathbf{E}_{\mathbf{0}} \exp (-i k \mathbf{r} \cdot \mathbf{s}) \sin \theta \mathrm{d} \theta \mathrm{d} \varphi .
$$

Here $\mathbf{r}=(r, \phi, z)$ denotes the polar coordinates at the focal area, $A$ is a constant value, $k$ is the wave-number, $\theta_{M}$ is the semi-aperture angle (related to the numerical aperture (NA) by means of $\left.\mathrm{NA}=\sin \theta_{M}\right), \theta$ and $\varphi$ are the coordinates at the Gaussian sphere of reference and the wave-front vector $\mathbf{s}$ reads $\mathbf{s}=(\sin \theta \cos \varphi, \sin \theta \sin \varphi, \cos \theta) . \mathbf{E}_{0}$ is the so-called vectorial angular spectrum, namely

$$
\mathbf{E}_{0}=\sqrt{\cos \theta}\left(f_{1} \mathbf{e}_{1}+f_{2} \mathbf{e}_{2}\right)=\sqrt{\cos \theta}\left(\left(\mathbf{E}_{i} \cdot \mathbf{e}_{1}\right) \mathbf{e}_{1}+\left(\mathbf{E}_{i} \cdot \mathbf{e}_{2}^{i}\right) \mathbf{e}_{2}\right)
$$


where $f_{1}$ and $f_{2}$ are the azimuthal and radial transverse components of the incident transverse field $\mathbf{E}_{i}$ respectively. Vectors $\mathbf{e}_{1}, \mathbf{e}_{2}$ and $\mathbf{e}_{2}^{i}$ are described by:

$$
\begin{array}{r}
\mathbf{e}_{1}(\varphi)=(-\sin \varphi, \cos \varphi, 0) \\
\mathbf{e}_{2}^{i}(\varphi)=(\cos \varphi, \sin \varphi, 0) \\
\mathbf{e}_{2}(\varphi, \theta)=(\cos \theta \cos \varphi, \cos \theta \sin \varphi,-\sin \theta) .
\end{array}
$$

Note that $\mathbf{e}_{1}, \mathbf{e}_{2}$ and $\mathbf{s}$ form a triad of mutually orthogonal right-handed system of unit vectors. Figure 1 summarizes the systems of coordinates used (a) at the entrance pupil, (b) at the Gaussian sphere of reference and (c) at the focal plane.

(a) Entrance pupil

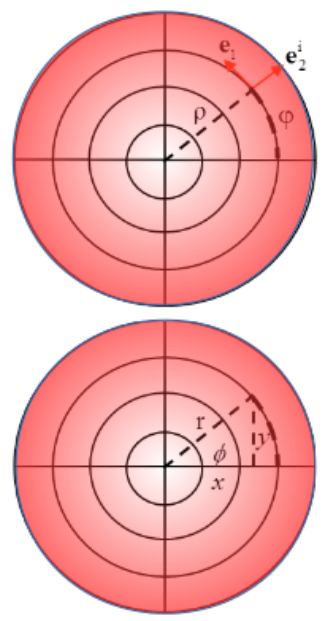

(b) Gaussian sphere of reference

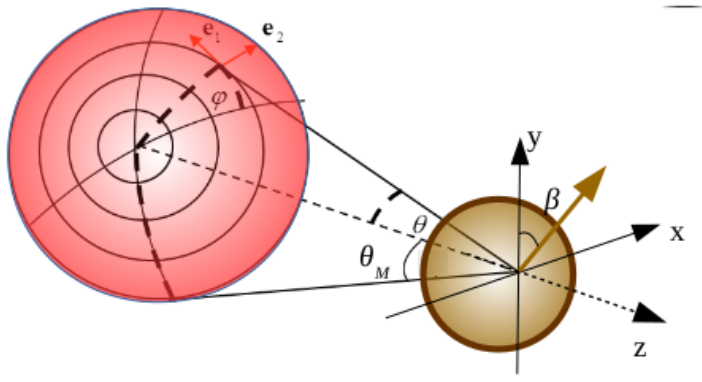

(c) Focal Plane

Figure 1: Coordinate system and geometrical magnitudes.

\section{3. Focused beams passing through polarizers.}

Linear polarizers have been described as a uniaxial anisotropic plane-parallel media of thickness $L$. The optical axis is assumed to be parallel to the plate surfaces and multiple internal reflexions are ignored[21, 22]. If the ordinary and extraordinary refractive indexes of the material are very similar, the incident and transmitted beams are related by

$$
\mathbf{P}_{\beta}\left[\mathbf{E}_{0}\right]=\exp \left(-i k \hat{n}_{o} L\right)\left(\mathbf{E}_{0} \cdot \mathbf{q}_{\mathbf{o}}\right) \mathbf{p}_{\mathbf{o}}+\exp \left(-i k \hat{n}_{e} L\right)\left(\mathbf{E}_{0} \cdot \mathbf{q}_{e}\right) \mathbf{p}_{e}
$$


where $\hat{n}_{o}=n_{o}-i \kappa_{o}$ and $\hat{n}_{e}=n_{e}-i \kappa_{e}$ are the ordinary and extraordinary complex refractive indices; $\mathbf{P}_{\beta}[]$ the linear operator that mathematically describes an ideal linear polarizer whose optical axis (c-axis) is described by $(\cos \beta, \sin \beta, 0)$. Thus, vectors $\mathbf{q}_{o}, \mathbf{p}_{o}, \mathbf{q}_{e}$ and, $\mathbf{p}_{e}$ are given by

$$
\begin{aligned}
& \mathbf{q}_{o}=t_{s} \cos \psi \mathbf{e}_{1}-t_{p} \sin \psi \mathbf{e}_{2} \\
& \mathbf{p}_{o}=t_{s}^{\prime} \cos \psi \mathbf{e}_{1}-t_{p}^{\prime} \sin \psi \mathbf{e}_{2} \\
& \mathbf{q}_{e}=t_{s} \sin \psi \mathbf{e}_{1}+t_{p} \cos \psi \mathbf{e}_{2} \\
& \mathbf{p}_{e}=t_{s}^{\prime} \sin \psi \mathbf{e}_{1}+t_{p}^{\prime} \cos \psi \mathbf{e}_{2}
\end{aligned}
$$

where $\cos \psi$ and $\sin \psi$ read

$$
\begin{aligned}
& \cos \psi=\frac{\cos \theta_{0} \cos (\varphi-\beta)}{\sqrt{1-\sin ^{2} \theta_{0} \cos ^{2}(\varphi-\beta)}} \\
& \sin \psi=-\frac{\sin (\varphi-\beta)}{\sqrt{1-\sin ^{2} \theta_{0} \cos ^{2}(\varphi-\beta)}} .
\end{aligned}
$$

The Fresnel transmission formulae for the first surface of the polarizing plate reads:

$$
\begin{aligned}
t_{s} & =\frac{2 \cos \theta}{\cos \theta+n_{o} \cos \theta_{0}} \\
t_{p} & =\frac{2 \cos \theta}{\cos \theta_{0}+n_{o} \cos \theta}
\end{aligned}
$$

where $\theta_{0}$ is the refraction angle, i.e. $\sin \theta=n_{o} \sin \theta_{0}$. The Fresnel coefficients for the second surface are:

$$
\begin{aligned}
t_{s}^{\prime} & =\frac{2 n_{o} \cos \theta_{0}}{\cos \theta+n_{o} \cos \theta_{0}} \\
t_{p}^{\prime} & =\frac{2 n_{o} \cos \theta_{0}}{\cos \theta_{0}+n_{o} \cos \theta} .
\end{aligned}
$$

O-type polarizers transmit ordinary waves and attenuates extraordinary ones, i.e. $\kappa_{o} \simeq 0$ and $\kappa_{e}>0$ and thus, $\mathbf{P}_{\beta}\left[\mathbf{E}_{0}\right]=\left(\mathbf{E}_{0} \cdot \mathbf{q}_{\mathbf{o}}\right) \mathbf{p}_{\mathbf{o}}$ and the polarizer axis direction is $(-\sin \beta, \cos \beta, 0)$. Accordingly, the electric field of a focused ${ }_{45}$ beam after the polarizer $\mathbf{P}_{\beta}[\mathbf{E}(\mathbf{r})]$ is obtained by projecting each contributions of the angular spectrum $\mathbf{P}_{\beta}\left[\mathbf{E}_{0}\right]$. In this case, the Richards-Wolf equation Eq.(1) reads [18]

$$
\begin{aligned}
\mathbf{P}_{\beta}[\mathbf{E}(\mathbf{r})] & =A \int_{0}^{\theta_{M}} \int_{0}^{2 \pi} \mathbf{P}_{\beta}\left[\mathbf{E}_{0}\right] \exp (-i k \mathbf{r} \cdot \mathbf{s}) \sin \theta \mathrm{d} \theta \mathrm{d} \varphi \\
& =A \int_{0}^{\theta_{M}} \int_{0}^{2 \pi}\left[\left(\mathbf{E}_{0} \cdot \mathbf{q}_{\mathbf{o}}\right) \mathbf{p}_{\mathbf{o}}\right] \exp (-i k \mathbf{r} \cdot \mathbf{s}) \sin \theta \mathrm{d} \theta \mathrm{d} \varphi
\end{aligned}
$$


Equation 9 clearly states that the field after the polarizer is non-uniform polarized and depends on the polarization state and topological charge of the vector 50 angular spectrum $\mathbf{E}_{0}$, and the NA of the focusing lens. In order to provide more insight on the meaning of the projected term $\mathbf{P}_{\beta}[\mathbf{E}(\mathbf{r})]$, it is straightforward to derive Eqs. 10 and 11(a-c) from Eqs. 9 and 5:

$$
\begin{aligned}
\mathbf{P}_{\beta}\left[\mathbf{E}_{0}\right] & =\left(\mathbf{E}_{0} \cdot \mathbf{q}_{\mathbf{o}}\right) \mathbf{p}_{\mathbf{o}}=\sqrt{\cos \theta}\left(f_{1} \mathbf{e}_{1}+f_{2} \mathbf{e}_{2}\right)\left(t_{s} \cos \psi \mathbf{e}_{1}-t_{p} \sin \psi \mathbf{e}_{2}\right) \mathbf{p} \\
& =\sqrt{\cos \theta}\left(f_{1} t_{s} \cos \psi-f_{2} t_{p} \sin \psi\right)\left(t_{s}^{\prime} \cos \psi \mathbf{e}_{1}-t_{p}^{\prime} \sin \psi \mathbf{e}_{2}\right)
\end{aligned}
$$

and thus,

$$
\begin{aligned}
& \mathbf{P}_{\beta}\left[\mathbf{E}_{0}\right]_{x}=\sqrt{\cos \theta}\left(f_{1} t_{s} \cos \psi-f_{2} t_{p} \sin \psi\right)\left(-t_{s}^{\prime} \cos \psi \sin \varphi-t_{p}^{\prime} \sin \psi \cos \theta \cos \varphi\right) \\
& \mathbf{P}_{\beta}\left[\mathbf{E}_{0}\right]_{y}=\sqrt{\cos \theta}\left(f_{1} t_{s} \cos \psi-f_{2} t_{p} \sin \psi\right)\left(t_{s}^{\prime} \cos \psi \cos \varphi-t_{p}^{\prime} \sin \psi \cos \theta \sin \varphi\right) \\
& \mathbf{P}_{\beta}\left[\mathbf{E}_{0}\right]_{z}=\sqrt{\cos \theta}\left(f_{1} t_{s} \cos \psi-f_{2} t_{p} \sin \psi\right) t_{p}^{\prime} \sin \psi \sin \theta
\end{aligned}
$$

Interestingly, Eq. 11c clearly shows that the beam after the polarizer displays a non-negligible longitudinal component even if the input beam is azimuthally polarized, i.e. when $f_{2}=0$. The components of the input beam $\mathbf{E}_{i}=\left(E_{i x}, E_{i y}, 0\right)$ of a spiral polarized beam fulfills the following equations:

$$
\begin{aligned}
& E_{i x}=E \cos (\varphi+\alpha) \\
& E_{i y}=E \sin (\varphi+\alpha) .
\end{aligned}
$$

as stated in [24]. Note that when $\alpha=0(\alpha=\pi / 2)$, radially (azimuthally) 55 polarized beams are obtained [25]. Now, we illustrate the behavior of Eqs. 10 and 11 for three values of $\alpha$ : 0 (radially polarized), $\pi / 4$ (spirally polarized) and $\pi / 2$ (azimuthally polarized). The polarization map of this kind of beams is shown in Fig. 2.

Figures 3(a) and 3(b) show the irradiance distributions of the focused electric field for a radially polarized beam before and after passing through a polarizer respectively. In what follows, the polarizer axis is set to the horizontal direction, i.e. $\beta=\pi / 2$. Moreover, the components images are normalized to the maximum value of the total irradiance $I_{T}$. The numerical aperture is set to NA=0.95. Figures display the irradiances of the $x$-component $I_{x}=\left|\mathbf{E}_{x}\right|^{2}, y$-component

${ }_{65} I_{y}=\left|\mathbf{E}_{y}\right|^{2}, z$-component $I_{z}=\left|\mathbf{E}_{z}\right|^{2}$, the transverse part $I_{t}=I_{x}+I_{y}$, the total field $I_{T}=I_{x}+I_{y}+I_{z}$ and the $3 \mathrm{D}$ polarization map. The polarization axis is set in the horizontal direction and thus, $I_{y}=0$ in Fig. 3(b). Since the irradiance of the longitudinal component is high, the total irradiance after the polarizer is quite different when compared with the $x$-component before the polarizer.

The same calculations have been carried out for an azimuthally polarized beam [see Fig. 4]. In agreement with Eq. $11(\mathrm{c})$ for $f_{2}=0$, it is worth to point 


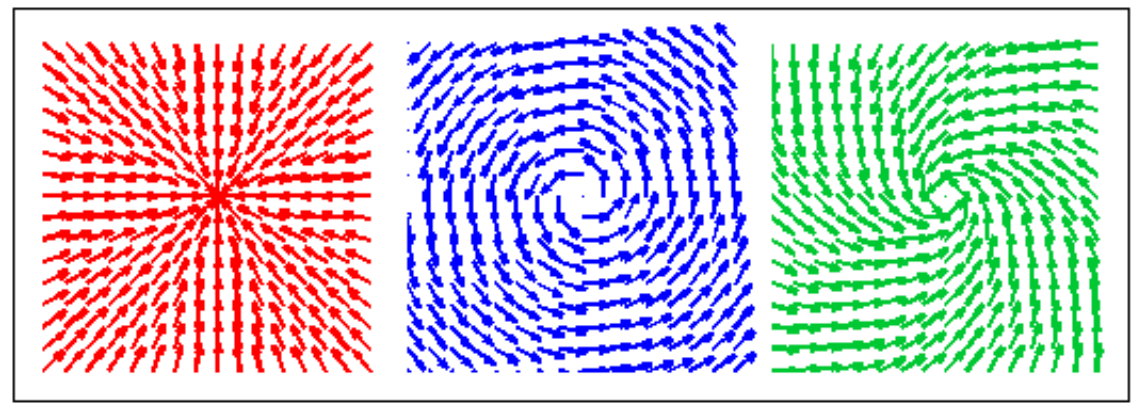

Figure 2: Polarization map: left: radial polarization, center: spiral polarization $\alpha=\pi / 4$, right: azimuthal polarization
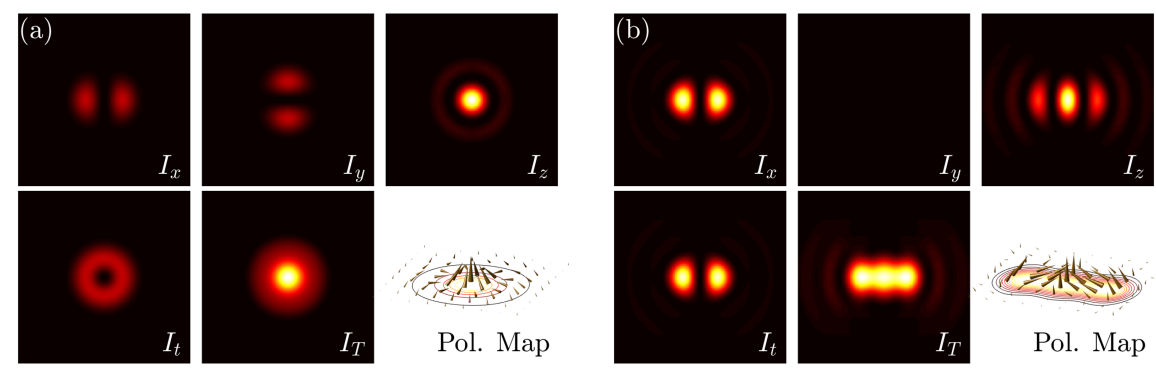

Pol. Map

Figure 3: Radially polarized beam NA=0.95, $\alpha=0$ : (a) before and (b) after the polarizer. Both subfigures display the following distributions: $I_{x}, I_{y}, I_{z}, I_{t}, I_{T}$, and the polarization map. Note that irradiance $I_{z}$ after the polarizer is weaker when compared with $I_{z}$ before the polarizer.

out that $I_{z} \neq 0$ after the polarizer. Finally, a spirally polarized beam with $\alpha=\pi / 4$ is also considered. Figs. 5(a) and 5(b) show the behavior of this field before and after the polarizers respectively.

\section{Analysis of the irradiance of the electric field after the polarizer}

In this section we introduce several parameters that may help to better understand the behavior of focused fields passing through a polarizer: (i) $\epsilon_{z}$ is the ratio of the integrated irradiance of the longitudinal component and the total integrated irradiance of the beam; (ii) $\tau$ is the ratio of the total inte-

80 grated irradiance of the polarized beam and the total integrated irradiance of the focused beam after the polarizer; (iii) $\beta_{z}$ is similar to $\epsilon_{z}$ but referred to the polarized beam; finally, (iv) $\tau_{z}$ relates the integrated irradiance of the longitudinal component of the projected beam and the total integrated irradiance of 

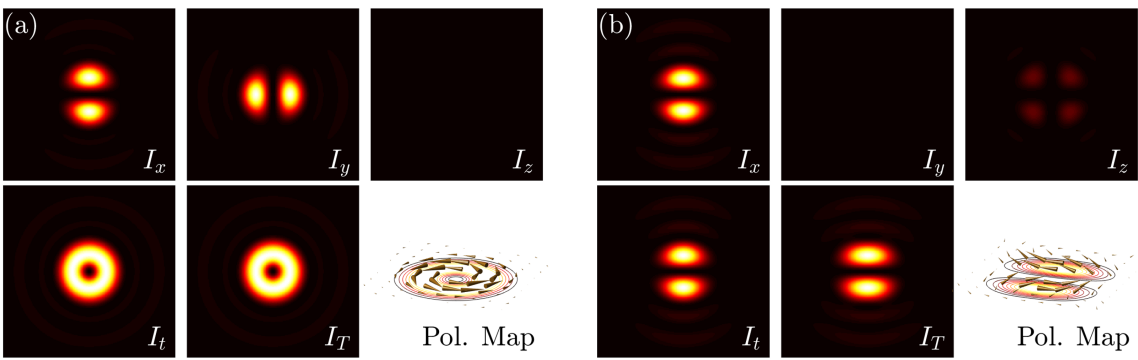

Figure 4: Azimuthally polarized beam NA=0.95, $\alpha=\pi / 2$ : (a) before and (b) after the polarizer. Both subfigures display the following distributions: $I_{x}, I_{y}, I_{z}, I_{t}, I_{T}$, and the polarization map. Note that irradiance $I_{z}$ after the polarizer is not zero.
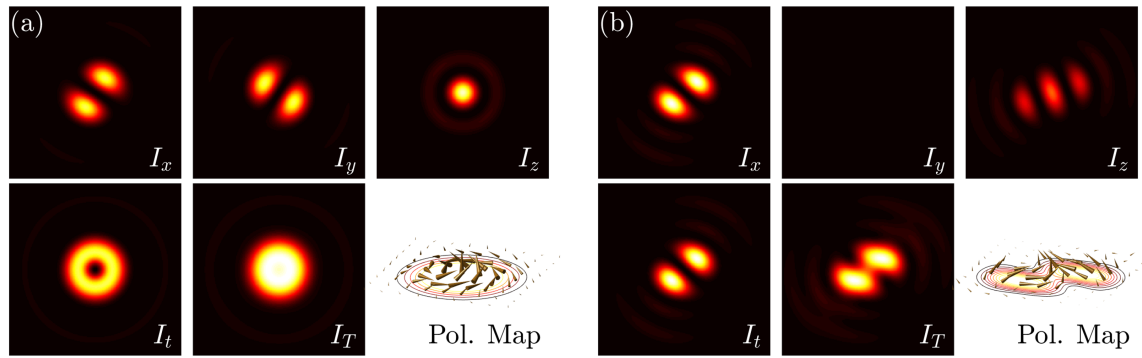

Figure 5: Spirally polarized beam NA=0.95, $\alpha=\pi / 4$ : (a) before and (b) after the polarizer. Both subfigures display the following distributions: $I_{x}, I_{y}, I_{z}, I_{t}, I_{T}$, and the polarization map.

the beam before the polarizer. Equations $13(\mathrm{a}-\mathrm{d})$ indicates how this parameters 85 are calculated.

$$
\begin{aligned}
\epsilon_{z} & =\frac{\iint\left|E_{z}(\mathbf{r})\right|^{2} \sin \phi d r d \phi}{\iint|\mathbf{E}(\mathbf{r})|^{2} \sin \phi d r d \phi} \\
\tau & =\frac{\iint\left|\mathbf{P}_{\beta}[\mathbf{E}(\mathbf{r})]\right|^{2} \sin \phi d r d \phi}{\iint|\mathbf{E}(\mathbf{r})|^{2} \sin \phi d r d \phi} \\
\beta_{z} & =\frac{\iint\left|\mathbf{P}_{\beta}[\mathbf{E}(\mathbf{r})]_{z}\right|^{2} \sin \phi d r d \phi}{\iint\left|\mathbf{P}_{\beta}[\mathbf{E}(\mathbf{r})]\right|^{2} \sin \phi d r d \phi} \\
\tau_{z} & =\frac{\iint\left|\mathbf{P}_{\beta}[\mathbf{E}(\mathbf{r})]_{z}\right|^{2} \sin \phi d r d \phi}{\iint|\mathbf{E}(\mathbf{r})|^{2} \sin \phi d r d \phi}
\end{aligned}
$$

Figure 6 shows the behavior of these parameters as a function of NA. As expected, $\epsilon_{z}$ increases with NA and is always zero for azimuthally polarized beams. $\tau$ compares the total energy of the transmitted and the incident beam: 

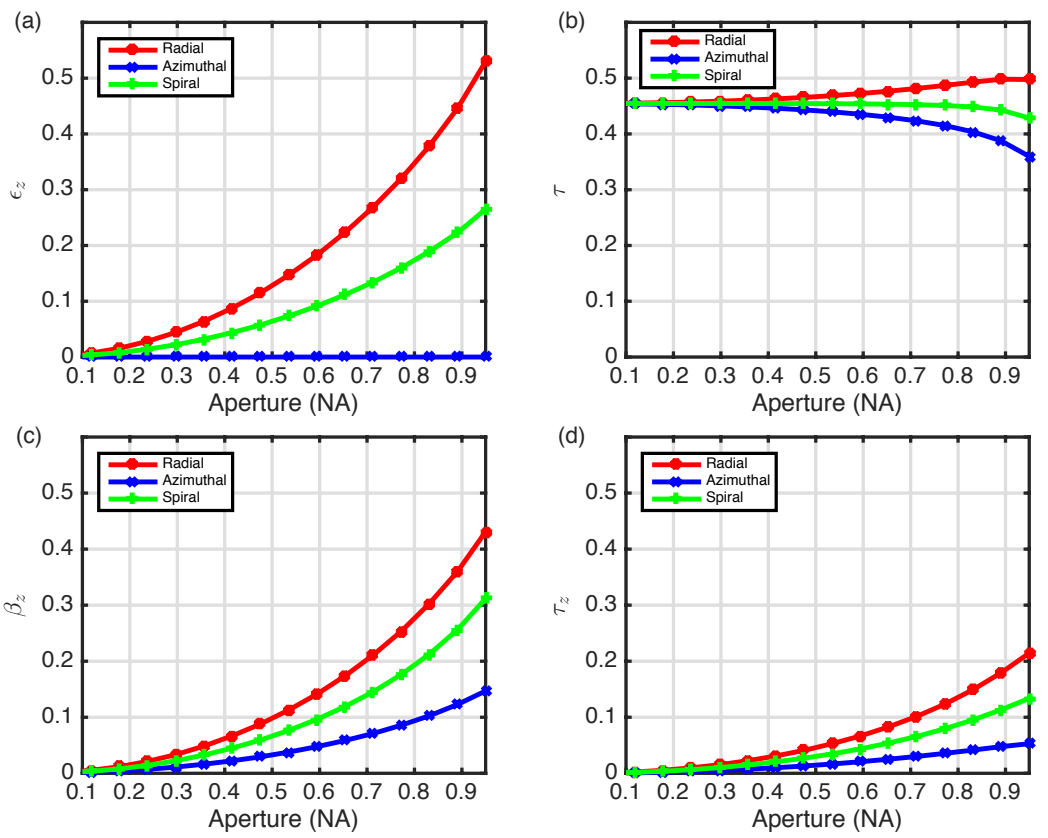

Figure 6: Behavior of the integrated irradiance parameters:(a) $\epsilon_{z}$ (b) $\tau,(\mathrm{c}) \beta_{z}$ and (d) $\tau_{z}$

this parameter ranges from 0.4 to 0.5 and displays an increasing or decreasing behavior depending on $\alpha$. Since the polarizer operator depends on the Fresnel formulae, the polarization direction of the beam plays a role in the interpretation of the behavior of this parameter. $\beta_{z}$ behaves in a similar way to $\epsilon_{z}$ but this parameter is related to the field after the polarizer; interestingly, $\beta_{z} \neq 0$ for azimuthally polarized beams. Moreover, the longitudinal component can be for the transmitted beam when compared with the impinging one: for instance $\epsilon_{z}(\mathrm{NA}=0.95, \alpha=\pi / 4)<\beta_{z}(\mathrm{NA}=0.95, \alpha=\pi / 4)$ (see Figs. $6(\mathrm{a})$ and $6(\mathrm{c}))$. At last, $\tau_{z}$ is similar to $\tau$ but referred to the $z$ - component. Note that the three curves considered increase with NA:

Finally, we introduce parameter $\rho$ described in Eq. 14. Note that $\rho$ is closely related to the similarity factor introduced in [26].

$$
\rho=\frac{\iint\left|\mathbf{P}_{\beta}[\mathbf{E}(\mathbf{r})]\right|\left|E_{x}(\mathbf{r})\right| \sin \phi d r d \phi}{\sqrt{\iint\left|\mathbf{P}_{\beta}[\mathbf{E}(\mathbf{r})]\right|^{2} \sin \phi d r d \phi} \sqrt{\iint\left|E_{x}(\mathbf{r})\right|^{2} \sin \phi d r d \phi}} .
$$

From the physical point of view, $\rho$ takes into account the correlation between the $x$-component of the field before the polarizer and the total amplitude after the polarizer. This parameter ranges from $0 \leq \rho \leq 1$; in particular, if $\rho=1$ then both distributions are indistinguishable. Figure 7 shows $\rho$ as a function of NA. Note that $\rho \approx 1$ for paraxial beams and displays a monotonically decreasing behavior with NA for the three cases considered. The minimum value for $\rho$ 


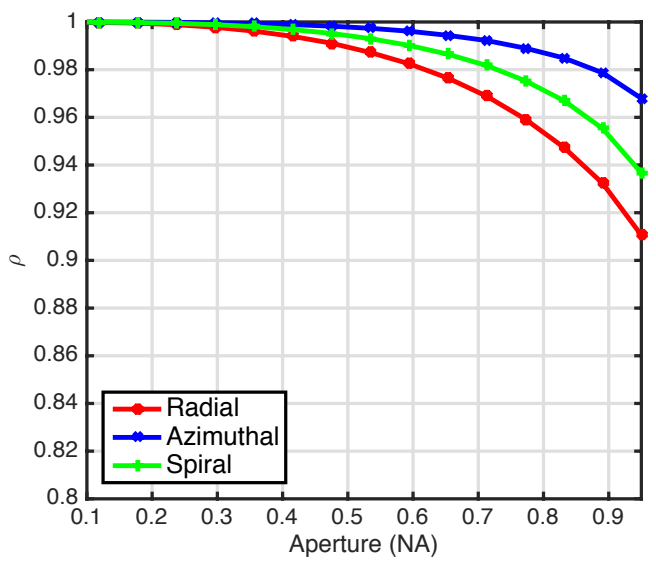

Figure 7: Behavior of the integrated irradiance parameter $\rho$

105 is obtained for radially polarized focused beams. These values are compatible with the results presented in Fig. 5(b). In particular, $I_{x}$ and $I_{T}$ looks quite different and consequently, a measure of the irradiance of a focused beam after a polarizer can produce an unappropriated estimation of the $x$-component.

\section{Concluding remarks}

110

In this paper we discussed how a spirally polarized focused beam is modified after passing through a linear polarizer. These devices are modeled as uniaxial anisotropic plane-parallel media with the optical axis parallel to the plate surfaces. Moreover, the electric field component in the direction of propagation and the Fresnel coefficients plays a key role in the description of this problem. Furthermore, the longitudinal irradiance can be larger for the transmitted beam when compared with the impinging one. Interestingly, pure-transverse azimuthal beams display a non-negligible longitudinal component after the polarizer.

It is worth to point out that the irradiance of the recorded beam after a polarizer differs from the projected component of the beam before passing the polarizer. The differences between these two distributions depend on the numerical aperture and the type of polarization. Despite these two distributions are identical within the paraxial domain, some differences exist with beams focused at high NA values (see Fig. 6). As a consequence, the evaluation of the irradiance of a focused beam after a polarizer can provide an unfair account of the projected component. On the other hand, the usual projector character of polarizers is recovered for paraxial beams when the influence of Fresnel coefficients can be neglected. 


\section{Acknowledgments}

130

This research is funded by Ministerio de Economia y Competitividad (MINECO) projects FIS2013-46475-C3-1-P, FIS2013-46475-C3-2-P and FIS2016-75147-C3$1-\mathrm{P}$.

\section{References}

[1] Y. Fainman, J. Shamir, Polarization of nonplanar wave fronts, App. Opt. 23 (18) (1984) 3188-3195.

[2] A. Aiello, C. Marquardt, G. Leuchs, Nonparaxial polarizers, Opt. Letters 34 (20) (2009) 3160-3162.

[3] J. Korger, T. Kolb, P. Banzer, A. Aiello, C. Wittmann, C. Marquardt, G. Leuchs, The polarization properties of a tilted polarizer, Opt. express 21 (22) (2013) 27032-27042.

[4] Q. Zhan, J. Leger, Focus shaping using cylindrical vector beams, Opt. Express 10 (7) (2002) 324-331.

[5] N. Davidson, N. Bokor, High-numerical-aperture focusing of radially polarized doughnut beams with a parabolic mirror and a flat diffractive lens, Opt. Lett. 29 (12) (2004) 1318-1320.

[6] Y. Kozawa, S. Sato, Sharper focal spot formed by higher-order radially polarized laser beams, J. Opt. Soc. Am. A 24 (6) (2007) 1793-1798.

[7] K. Lindfors, A. Priimagi, T. Setälä, A. Shevchenko, A. T. Friberg, M. Kaivola, Local polarization of tightly focused unpolarized light, Nat. Photon. 1 (4) (2007) 228-231.

[8] Q. Zhan, Cylindrical vector beams: from mathematical concepts to applications, Adv. Opt. Photon. 1 (1) (2009) 1-57.

[9] D. Maluenda, I. Juvells, R. Martínez-Herrero, A. Carnicer, Reconfigurable beams with arbitrary polarization and shape distributions at a given plane, Opt. Express 21 (5) (2013) 5432-5439.

[10] K. A. Serrels, E. Ramsay, R. J. Warburton, D. T. Reid, Nanoscale optical microscopy in the vectorial focusing regime, Nat. Photon. 2 (5) (2008) 311314.

[11] H. Wang, L. Shi, B. Lukyanchuk, C. Sheppard, C. T. Chong, Creation of a needle of longitudinally polarized light in vacuum using binary optics, Nat. Photon. 2 (8) (2008) 501-505.

[12] L. Yang, X. Xie, S. Wang, J. Zhou, Minimized spot of annular radially polarized focusing beam, Opt. Letters 38 (8) (2013) 1331-1333. 
[13] Y. Zha, J. Wei, H. Wang, F. Gan, Creation of an ultra-long depth of focus super-resolution longitudinally polarized beam with a ternary optical element, J. Optics 15 (7) (2013) 075703.

[14] D. Maluenda, R. Martínez-Herrero, I. Juvells, A. Carnicer, Synthesis of highly focused fields with circular polarization at any transverse plane, Opt. Express 22 (6) (2014) 6859-6867. doi:10.1364/0E.22.006859.

[15] R. Martínez-Herrero, I. Juvells, A. Carnicer, Design of highly focused fields that remain unpolarized on axis, Opt. Letters 39 (20) (2014) 6025-6028.

[16] R. Martínez-Herrero, D. Maluenda, I. Juvells, A. Carnicer, Experimental implementation of tightly focused beams with unpolarized transversal component at any plane, Opt. Express 22 (26) (2014) 32419-32428.

[17] A. Carnicer, I. Juvells, B. Javidi, R. Martínez-Herrero, Optical encryption in the longitudinal domain of focused fields, Opt. Express 24 (7) (2016) 6793-6801.

[18] R. Martínez-Herrero, D. Maluenda, I. Juvells, A. Carnicer, Polarisers in the focal domain: Theoretical model and experimental validation., Sci. Rep. 7 (2017) 42122.

[19] P. Yeh, Electromagnetic propagation in birefringent layered media, J. Opt. Soc. Am. 69 (5) (1979) 742-756.

[20] P. Yeh, Optics of anisotropic layered media: a new $4 \times 4$ matrix algebra, Surf. Sci. 96 (1) (1980) 41-53.

185 [21] P. Yeh, Extended Jones matrix method, J. Opt. Soc. Am. 72 (4) (1982) 507-513.

[22] C. Gu, P. Yeh, Extended Jones matrix method II, J. Opt. Soc. Am. A 10 (5) (1993) 966-973.

[23] E. Wolf, Electromagnetic diffraction in optical systems. I. An integral representation of the image field, P. Roy. Soc. London A Mat. 253 (1274) (1959) 349-357.

[24] F. Gori, Polarization basis for vortex beams, JOSA A 18 (7) (2001) 16121617.

[25] R. Martínez-Herrero, P. Mejías, Propagation of light fields with radial or azimuthal polarization distribution at a transverse plane, Optics express 16 (12) (2008) 9021-9033.

[26] R. Martınez-Herrero, I. Juvells, A. Carnicer, On the physical realizability of highly focused electromagnetic field distributions, Opt. Lett. 38 (12) (2013) 2065-2067. 